\title{
SPP1 and FN1 are significant gene biomarkers of tongue squamous cell carcinoma
}

\author{
XIAO-LIANG XU ${ }^{1}$, HUI LIU ${ }^{2}$, YING ZHANG ${ }^{3}$, SU-XIN ZHANG $^{4}$, ZHONG CHEN $^{4}$, YANG BAO $^{4}$ and TIAN-KE LI ${ }^{4}$ \\ ${ }^{1}$ Department of Stomatology, The Second Hospital of Tangshan City; ${ }^{2}$ Department of Stomatology, North China University of \\ Science And Technology Affiliated Hospital, Tangshan, Hebei 063000; ${ }^{3}$ Department of Stomatology, \\ The Third Hospital of Shijiazhuang City; ${ }^{4}$ Department of Stomatology, \\ The Fourth Hospital of Hebei Medical University, Shijiazhuang, Hebei 050011, P.R. China
}

Received November 13, 2019; Accepted September 18, 2020

DOI: $10.3892 / 01.2021 .12974$

\begin{abstract}
Tongue squamous cell carcinoma (TSCC) is one of the most common malignant tumor types in the oral and maxillofacial region. The etiology and pathogenesis behind TSCC is complicated. In the present study, three gene expression profiles, namely GSE31056, GSE13601 and GSE78060, were downloaded from the Gene Expression Omnibus (GEO). The GEO2R online tool was utilized to identify differentially expressed genes (DEGs) between TSCC and normal tissue samples. Furthermore, a protein-protein interaction (PPI) network was constructed and hub genes were validated and analyzed. A total of 83 common DEGs were obtained in three datasets, including 48 upregulated and 35 downregulated genes. Pathway enrichment analysis indicated that DEGs were primarily enriched in cell adhesion, extracellular matrix (ECM) organization, and proteolysis. A total of 63 nodes and 218 edges were included in the PPI network. The top 11 candidate hub genes were acquired, namely plasminogen activator urokinase (PLAU), signal transducer and activator of transcription 1, C-X-C motif chemokine ligand 12, matrix metallopeptidase (MMP) 13, secreted phosphoprotein 1 (SPP1), periostin, MMP1, MMP3, fibronectin 1 (FN1), serpin family $E$ member 1 and snail family transcriptional repressor 2. Overall, 83 DEGs and 11 hub genes were screened from TSCC and normal individuals using bioinformatics and microarray technology. These genes may be used as diagnostic and therapeutic biomarkers for TSCC. In addition, SPP1 and $\mathrm{FNl}$ were identified as potential biomarkers for the progression of TSCC.
\end{abstract}

Correspondence to: Dr Tian-Ke Li, Department of Stomatology, The Fourth Hospital of Hebei Medical University, 12 Jiankang Road, Shijiazhuang, Hebei 050011, P.R. China

E-mail: litianb112@163.com

Key words: tongue squamous cell carcinoma, microarray technology, secreted phosphoprotein 1 , fibronectin 1 , hub genes

\section{Introduction}

Tongue cancer is a common malignant tumor type of the oral and maxillofacial cavity (1). The incidence of tongue carcinoma in males is higher than that of females and its most common histopathological feature is squamous cell carcinoma, usually located in the anterior two thirds of the tongue (2). Tongue adenocarcinoma is a rare distinct clinicopathological entity of tongue cancer and is mostly located in the root of the tongue (1). Furthermore, lymphatic epithelial cancer and undifferentiated carcinoma may sometimes occur in the root of the tongue (1). Tongue squamous cell carcinoma (TSCC) accounts for $\sim 50-60 \%$ of oral malignancies and $0.8-2 \%$ of systemic malignancies $(3,4)$. TSCC is highly malignant, invasive and prone to lymph node metastasis (5). The etiology and pathogenesis of TSCC are complicated (6,7). Standard treatment for TSCC includes surgical resection; assisted positive surgical margin and vein detection; lymphatic or nerve dissection; postoperative radiotherapy and chemotherapy (8). Although substantial progress has been made in the diagnosis and treatment, the 5-year survival rate for patients with TSCC remains low due to an increased risk of recurrence and lymph node metastasis (9). Therefore, TSCC poses a serious threat to human health (10).

The etiology of TSCC is yet to be fully understood. However, several common environmental risk factors have been associated with TSCC, including heat, chronic damage, ultraviolet radiation, $\mathrm{X}$-rays and other radioactive carcinogenic substances $(11,12)$. For example, tongue and buccal mucosa cancer may occur following chronic irritation in areas such as residual roots, sharp cusps and tooth prosthetics (13). In addition, neuropsychiatric, endocrine and genetic factors, as well as the immune system status have been implicated in the development of TSCC (14). Recently, an increasing number of studies have investigated the changes in gene expression associated with TSCC (15-17). The occurrence of TSCC has been associated with repeated damage, hyperemia and proliferation of tongue mucosa cells, which are caused by several factors (18). Furthermore, tongue cancer is triggered by a gradual increase in tongue mucosa cell metabolism, thus resulting in repeated DNA breaks and recombination (19). Recently, several studies have investigated the differentially 
expressed genes (DEGs) in patients with TSCC, as well as their roles in various signaling pathways, molecular functions and biological processes, using bioinformatics analysis (20-22). Usami et al (23) found that intercellular adhesion molecule 1 plays an important role in the development of tongue cancer through promoting cancer cell proliferation, angiogenesis, lymphatic vessel density and adhesion of giant cells. In addition, Zhang et al (24) demonstrated that galectin-3 regulates the Wnt/ $\beta$-catenin signaling pathway and Akt phosphorylation in vitro, thereby mediating cancer cell migration and invasion, and resulting in tongue cancer progression. Wang et al (25) also showed that enhancer of zeste 2 polycomb repressive complex 2 subunit expression is associated with the neoplasm staging and its overexpression increases the risk of tongue cancer. Furthermore microarray expression datasets have been increasingly used to identify novel microRNA (miRNA) biomarkers with diagnostic and prognostic value in oral cancer and other types of cancer (20,26-30).

TSCC is one of the most common types of head and neck malignant tumors and the most common cancer in the oral cavity (31). Tongue cancer can be divided into two types according to the anatomic location of the tumor: Oral tongue cancer, which occurs in the anterior two thirds of the tongue; and tongue base cancer, which involves the posterior third of the tongue (15). The incidence of oral tongue carcinoma is higher than that of tongue base carcinoma (32). The majority of tongue cancers, and especially oral tongue carcinomas, are derived from moderately or highly differentiated squamous epithelial cells (33). However, adenocarcinomas, lymphatic epithelial cancer and undifferentiated carcinomas are relatively rare and are mostly derived from the base of the tongue (34). In addition, small salivary gland-derived malignant tumors, such as adenoid cystic carcinoma have also been identified as a type of tongue cancer. Treatment strategies for tongue cancer include simple surgery; radiation therapy, including external irradiation and inter-plant insertion brachytherapy; systemic chemotherapy; and targeted therapy $(35,36)$. As a deeper understanding of the molecular pathways involved in tongue cancer has been achieved, the discovery of novel and promising targets for cancer treatment is increasing (37). As such, exploring the exact molecular mechanisms of action, as well as reliable therapeutic targets for TSCC has attracted wide attention (15). With the development of gene sequencing technology, a large number of DEGs have been identified in various tumor types $(38,39)$. DEGs play several roles in the occurrence and development of various diseases, including transcriptional regulation, post-transcriptional processing and regulation of protein expression. The present study hypothesized that DEGs could also play a key role in the development of TSCC and in its malignant progression, thus serving as molecular markers and therapeutic targets for TSCC.

Microarray technology allows the simultaneous analysis of changes in the expression levels of multiple genes to obtain gene sets that may be involved in TSCC (21). DEGs have been associated with the tumor grade and prognosis for patients with TSCC (40). The expression of key molecular markers may be used as independent prognostic factors; therefore, further in-depth studies should be conducted to investigate the potential mechanisms of action behind abnormally expressed genes. These markers may affect the initiation and malignant progression of TSCC and may be used as therapeutic targets (41). Therefore, there is an urgent need to detect and analyze reliable target genes for TSCC.

\section{Materials and methods}

Microarray data. A total of three gene expression profiles, namely GSE31056, GSE13601 and GSE78060, were retrieved from the Gene Expression Omnibus (GEO; www.ncbi.nlm.nih. gov/geo) dataset. The GSE31056 microarray data consisted of 22 TSCC and 24 normal tissue samples. In addition, the mRNA expression profile of 31 patients with TSCC and 26 healthy individuals was obtained from GSE13601. Similarly, a total of 27 TSCC and three normal tissue samples were available in the GSE78060 dataset.

Identification of DEGs. GEO2R, an online tool (https://www. ncbi.nlm.nih.gov/geo/geo2r/), was utilized to identify DEGs from the GEO series between TSCC and normal tissues. Absent and duplicate probe sets were excluded. The cut-off points were set to $\mid \log _{2} \mathrm{FCl}>1.5$ and adjusted $\mathrm{P}<0.05$. The fold change indicated expression in TSCC tissue samples/expression in normal tissue samples. Subsequently, DEGs were visualized using volcano plots and heatmaps using R software (version 3.5.3; The R Foundation) and Functional Enrichment analysis tool (Funrich; version 3.1.3; http://funrich.org/index. html; FunRich Co. Ltd.). A Venn diagram intersected all three datasets was constructed to acquire the common DEGs.

Functional enrichment analysis of DEGs. The Database for Annotation, Visualization and Integrated Discovery (DAVID; version 6.8; https://david.ncifcrf.gov/; DAVID Bioinformatics Forum), an open online platform, was utilized to elucidate the potential biological meaning of DEGs using Gene Ontology (GO) and Kyoto Encyclopedia of Genes and Genomes (KEGG) pathway enrichment analyses. GO analysis of DEGs was carried out from three main aspects of biological information, namely biological process (BP), molecular function (MF) and cellular component (CC). $\mathrm{P}<0.05$ was considered to indicate a statistically significant difference. Subsequently, the results were visualized using the ggplot package of R software (version 3.5.3; The R Foundation).

Construction of the protein-protein interaction (PPI) network and module analysis. The PPI network was constructed using the Search Tool for the Retrieval of Interacting Genes database (STRING; https://string-db.org) and was visualized using the Cytoscape software (version 3.7.1; https://cytoscape. org/what_is_cytoscape.html). Molecular Complex Detection within the Cytoscape software (version 3.7.1, https://cytoscape. org/what_is_cytoscape.html) was then applied to screen significant modules in the PPI network. The criteria default parameters were as follows: Degree cut-off $=10, \mathrm{k}$-core $=2$, node score cut-off $=0.2$ and max. depth $=100$. Subsequently, the cBioportal database (www.cbioportal.org), an online tool integrating the International Cancer Genome Consortium (icgc.org), the Cancer Genome Atlas (portal.gdc.cancer.gov) and other cancer genome databases, was utilized to construct the co-expression network of hub genes in the module. Finally, the results of the biological process analysis and co-expression 
Table I. Summary of tongue squamous cell carcinoma microarray datasets.

\begin{tabular}{llll}
\hline Series & Platform & \multicolumn{1}{c}{ GeneChip } & Samples \\
\hline GSE31056 & GPL10526 & Affymetrix Human Genome U133 Plus 2 Array & 96 \\
GSE13601 & GPL8300 & Affymetrix Human Genome U95 Version 2 Array & 58 \\
GSE78060 & GPL570 & Affymetrix Human Genome U133 Plus 2 Array & 30 \\
\hline
\end{tabular}

A

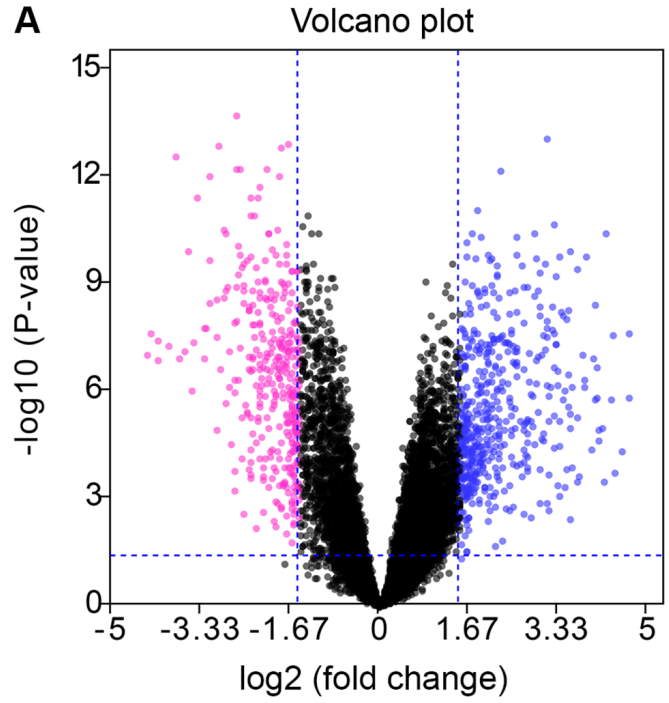

C

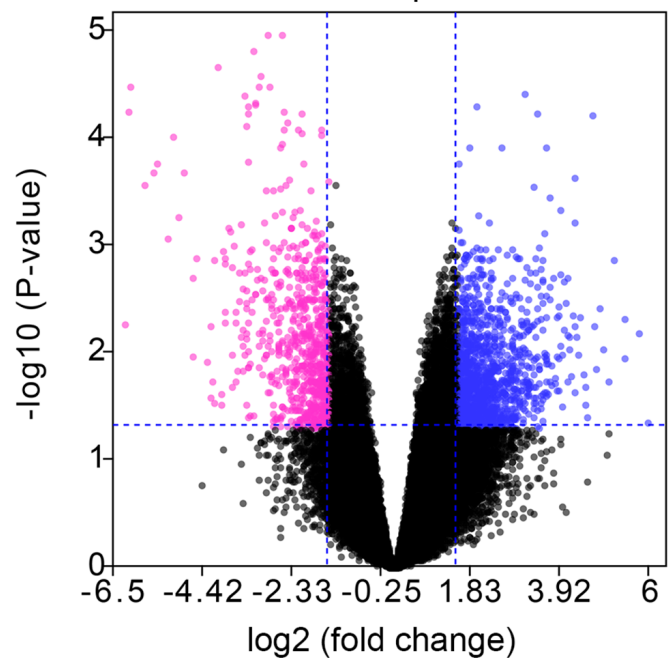

B

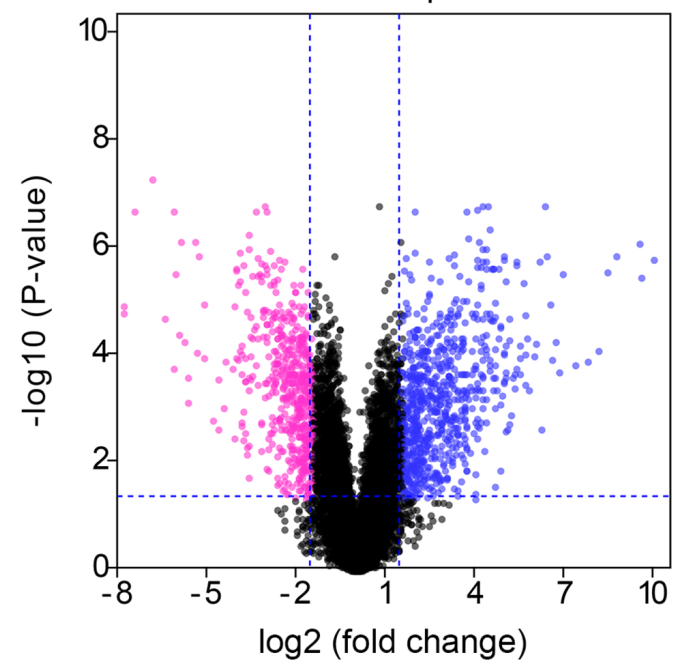

D

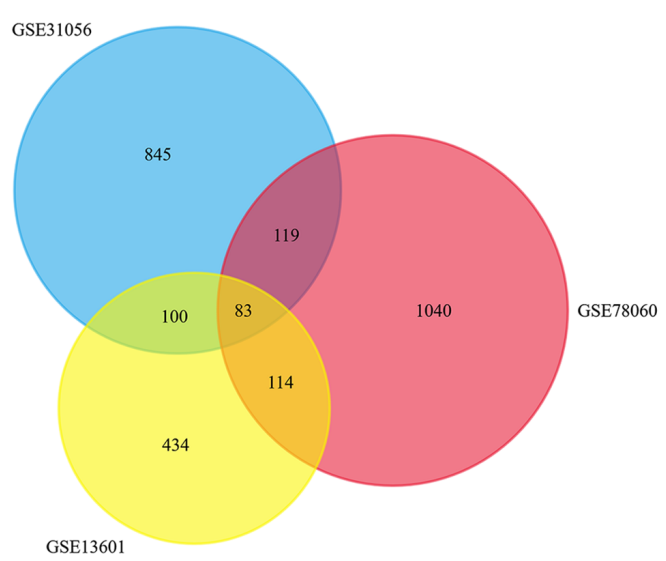

Figure 1. Volcano plots and a Venn diagram of DEGs in tongue squamous cell carcinoma samples. DEGs were selected with $\mid \log _{2} \mathrm{FCl}>1.5$ and adj. $\mathrm{P}<0.05$ among the mRNA expression profiling sets, (A) GSE13601, (B) GSE31056 and (C) GSE78060. (D) The 3 datasets showed an overlap of 83 genes. DEG, differentially expressed gene.

network of hub genes were visualized using the BiNGO tool in Cytoscape software (version 3.7.1, https://cytoscape. org/what_is_cytoscape.html).

Validation and analysis of hub genes. Published microarray data were retrieved from the Oncomine database (http://www. oncomine.org) to validate the expression levels of hub genes in TSCC tissues. Subsequently, survival curves were drawn to evaluate the prognostic significance of hub genes using the Kaplan-Meier plotter database (http://kmplot.com/analysis/). Finally, the Gene Expression Profiling Interactive Analysis database was used to assess the differential expression of several hub genes in each TNM stage (42). Independent-samples T test was applied to identify statistical differences.

Prediction and enrichment analysis of miRNAs related to hub genes. Targetscan (www.targetscan.org), an online database that predicts potential interactions between genes and miRNAs, was used to predict miRNAs associated with hub genes. Subsequently, enrichment analysis of the predicted miRNAs was performed with the DNA Intelligent Analysis (DIANA)-miRPath software (version 3.0; http://diana.imis. 
Table II. Screening of differentially expressed genes in oral squamous cell carcinoma samples.

DEGs

List of gene symbols

Upregulated DEGs

MMP1, TYMP, MMP10, KRT16, MMP13, SPP1, MMP3, LAMC2, IFI27, PTHLH, RBP1, ISG15, MMP12, TNC, TGFBI, CXCL11, FSCN1, MYO1B, SERPINE1, STAT1, CDH3, ITGA6, POSTN, SNAI2, PLAU, LAMA3, FAP, XAF1, DUSP14, APOL1, COL5A2, RSAD2, TP63, MYO10, F2RL1, PTK7, ACTN1, LAMB3, EXT1, IFIT3, FN1, IGFBP3, ITGA3, DFNA5, IFI44, COL4A1, LOXL2, MICAL2

Downregulated DEGs PDPN, RECK, DPT, CFD, IFI6, MEOX2, CXCL12, ABCA6, NR3C2, ITM2A, BEX4, PBX1, GDF10, CBX7, MYRIP, LIFR, CLU, SLITRK5, LPIN1, GPRASP1, KAT2B, CDO1, GATM, GPC3, SORBS2, FRZB, METTL7A, CILP, RNASE4, DLK1, CRISP3, MFAP4, ALDH1A1, SELENBP1, ADH1B

DEG, differentially expressed genes.
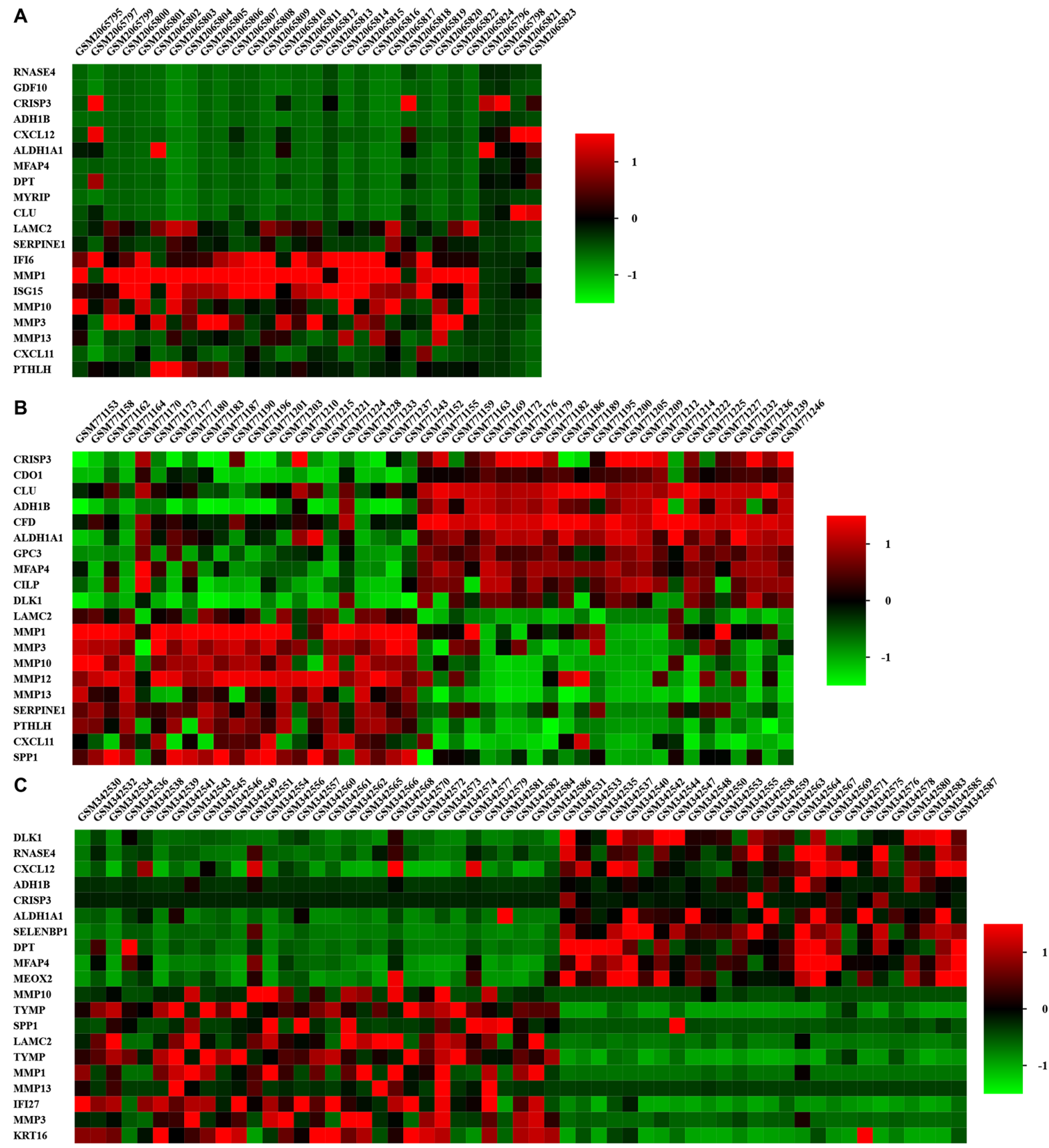

Figure 2. Heatmaps of the DEGs identified between tongue squamous cell carcinoma tissues and normal samples. DEGs identified from the (A) GSE78060 (B) GSE31056 and (C) DEGs GSE13601 databases. DEG, differentially expressed gene. 

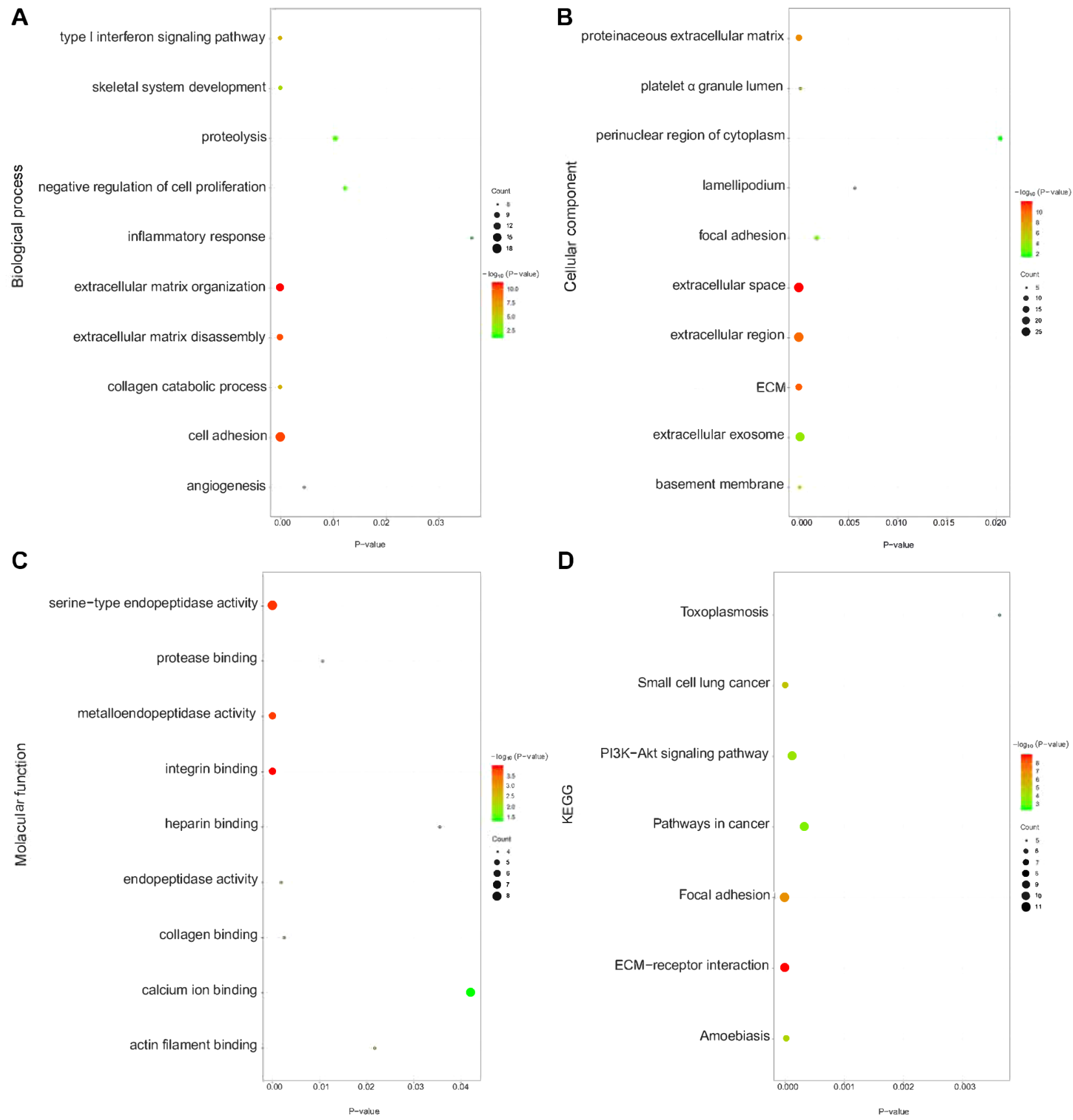

Figure 3. Enrichment analysis of DEGs in tongue squamous cell carcinoma. Enrichment analyses of the (A) biological process, (B) cellular component, (C) molecular function and (D) KEGG pathway of the DEGs. DEG, differentially expressed gene; ECM, extracellular matrix.

athena-innovation.gr/DianaTools/index.php?r=mirpath/index; DIANA LAB, University of Thessaly), a handy online tool for enrichment analysis.

Reverse transcription-quantitative polymerase chain reaction (RT-qPCR) assay. A total of eight individuals were recruited, including four healthy controls and four patients with TSCC. Following surgery, four TSCC tissue samples from four patients with TSCC and four para-carcinoma tissues were obtained. The research conformed to the standards set by the Declaration of Helsinki and was authorized by the Human Ethics and Research Ethics Committees of the Fourth Hospital of the Hebei Medical University. Written informed consents were obtained from all participants.
Total RNA was extracted from the tissue samples using the TRIzol ${ }^{\circledR}$ (Beijing Biolab Technology Co., Ltd.) and reverse transcribed into cDNA with the Servicebio ${ }^{\circledR}$ RT First Strand cDNA Synthesis kit (cat. no. G3330; Wuhan Servicebio Biotechnology Co., Ltd.) for $60 \mathrm{~min}$ at $42^{\circ} \mathrm{C}$. Terminate the reaction by heating at $70^{\circ} \mathrm{C}$ for $5 \mathrm{~min}$. RT-qPCR was performed in a Light Cycler ${ }^{\circledR} 4800$ System (Roche Diagnostics) with a specific set of primers for the amplification of secreted phosphoprotein 1 (SPP1) and fibronectin 1 (FN1) genes. Primers used were as follows: SPP1 forward, 5'-CTAAACCCTGAC CCATCT-3', reverse, 5'-CAATGCCTTCTTTCATCT-3'; GAPDH forward, 5'-ATCCGATTACCGATACCTAGACC-3', reverse, 5'-ATGGACTATATCCGACGACGA-3'; and FN1 forward, 5'-CCAACTACCAGTAGCGAAAA -3', reverse, 


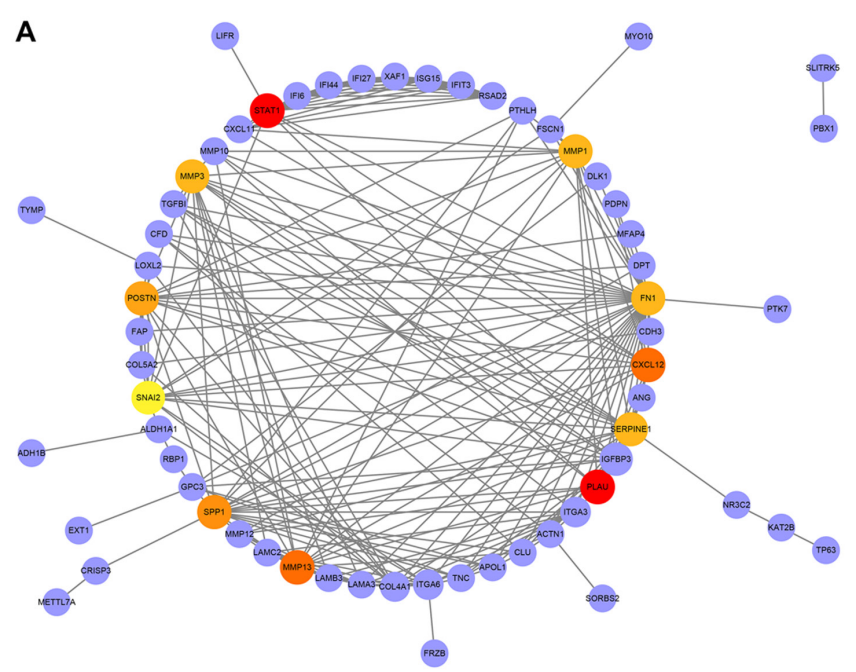

B

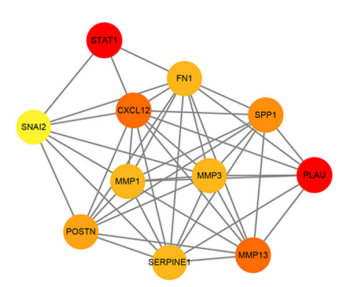

Figure 4. PPI network and hub genes in tongue squamous cell carcinoma. (A) The PPI network of DEGs contained 63 nodes and 218 edges. The depth of color and the size of node represents its significance. The brighter the color and the larger the diameter, the more significant the node is. (B) The most significant module was obtained from PPI network of DEGs. DEG, differentially expressed gene; PPI, protein-protein interaction.

5'-GCAGGGAAAGGAAAGAAA-3'. The thermocycling conditions used were as follows: $95^{\circ} \mathrm{C}$ for $15 \mathrm{sec}$ followed by 30 cycles of $60^{\circ} \mathrm{C}$ for $60 \mathrm{sec}$. The relative quantification units (relative quantification $=2^{-\Delta \Delta \mathrm{Cq}}$, where $\mathrm{Cq}$ represents quantification cycle values) of each sample were calculated (43) and presented as fold change of gene expression relative to the control group. GAPDH was used as an endogenous control.

Statistics. The data was expressed as percentage of the total and the mean $\pm \mathrm{SD}$. When two groups were compared, the paired Student's t-test were used to determine statistical significance. For the stage analysis, one-way ANOVA was used to compare DEG expression levels, using the pathological stage as a variable for calculating differential expression. All statistical analyses were conducted using SPSS software, version 23.0 (IBM Corp.). A P-value $<0.05$ was considered statistically significant.

\section{Results}

Identification of DEGs in TSCC. A total of 1,147, 731 and 1,356 DEGs were obtained from the GSE31056, GSE13601 and GSE78060 datasets, respectively. All datasets were downloaded from the GEO database (Table I). Identified DEGs were visualized using volcano plots and heatmaps (Figs. 1A-C and 2). DEGs were selected with $\mid \log 2 \mathrm{FCl}>1.5$ and adj. P-value $<0.05$ among the mRNA expression profiling sets in GSE13601 (Fig. 1A), GSE31056 (Fig. 1B), and GSE78060 (Fig. 1C). The Venn diagram demonstrated that 83 common

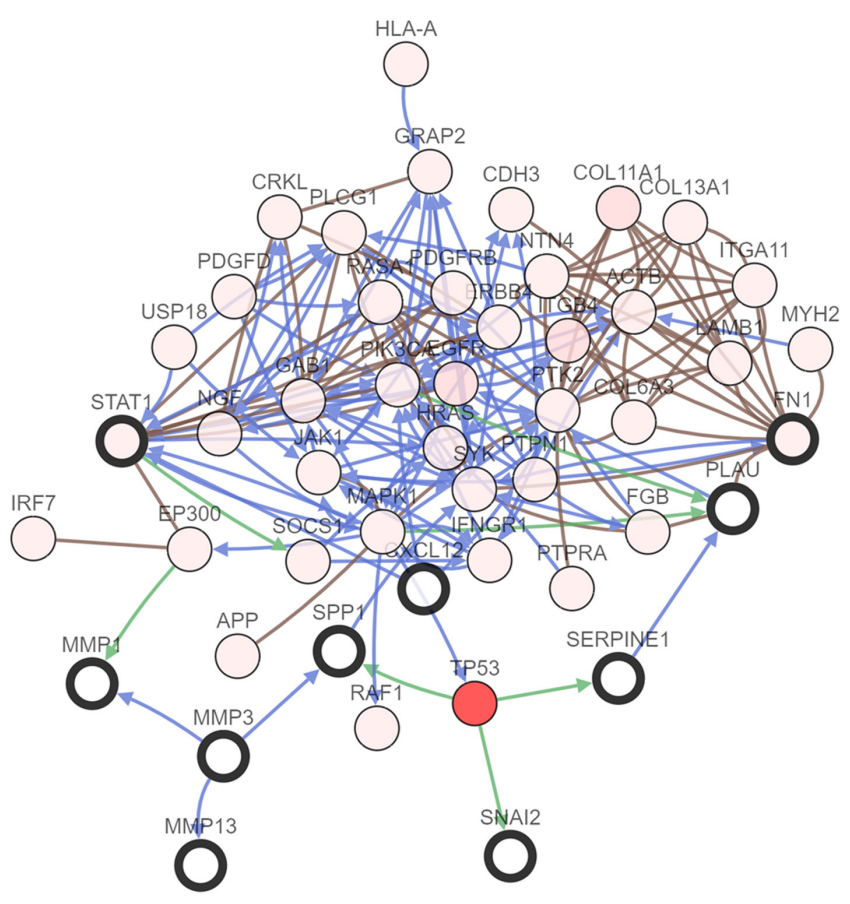

Figure 5. Hub genes and their co-expression genes were analyzed using cBioPortal. Nodes with bold black outline represent hub genes. Nodes with thin black outline represent the co-expression genes. The green arrows indicate the strongest correlations between nodes, the blue arrows the second and the brown lines the weakest.

DEGs were obtained from the three datasets, including 48 upregulated and 35 downregulated genes (Fig. 1D; Table II).

Enrichment analysis for DEGs. The enrichment analysis indicated that DEGs were mainly enriched in BPs associated with cell adhesion, ECM organization, ECM disassembly and proteolysis. With respect to CC, DEGs were primarily enriched in the extracellular space, extracellular region, extracellular exosome and ECM. DEGs were mainly associated with integrin binding, serine-type endopeptidase activity and metalloendopeptidase activity in the MF category. KEGG pathway enrichment analysis found that DEGs were remarkably enriched in the PI3K-Akt signaling pathway, focal adhesion and ECM-receptor interaction (Fig. 3).

PPI network construction and module analysis. To further understand the association among DEGs, a PPI interaction network was conducted, including 63 nodes and 218 edges (Fig. 4A). Subsequently, the most significant module in the PPI network was selected. The top 11 candidate hub genes were also selected, namely plasminogen activator urokinase (PLAU), signal transducer and activator of transcription 1 (STAT1), C-X-C motif chemokine ligand 12 (CXCL12), matrix metallopeptidase (MMP) 13, SPP1, periostin, MMP1, MMP3, FN1, serpin family E member 1 (SERPINE1) and snail family transcriptional repressor 2 (SNAI2). The most significant module was obtained from PPI network of DEGs (Fig. 4B).

Hub genes and their co-expression genes were analyzed using cBioPortal. Nodes with bold black outline represent hub genes. Nodes with thin black outline represent the co-expression genes. Subsequently, the co-expression network of the 11 hub genes was constructed using the cBioportal online 


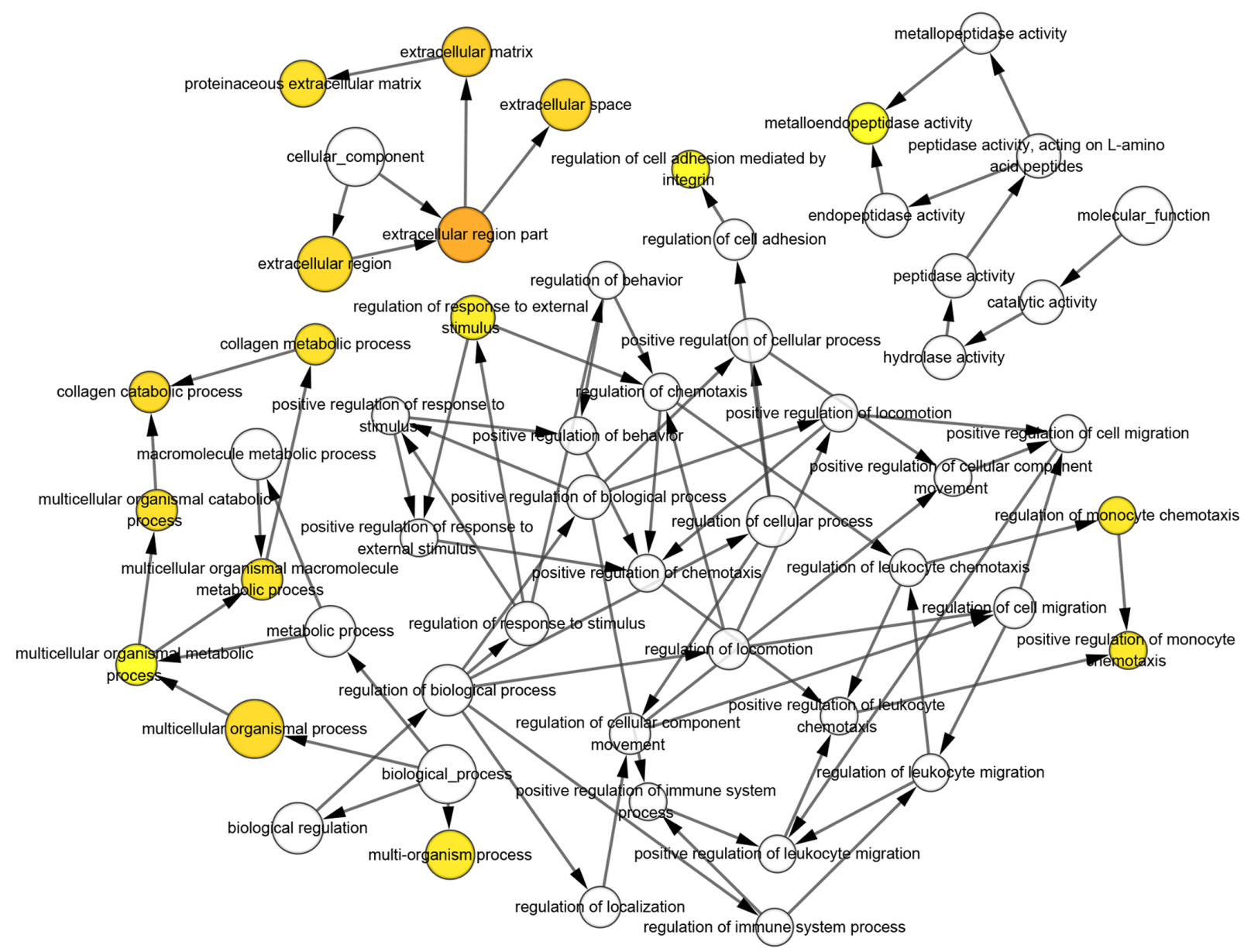

Figure 6. The biological process analysis of hub genes was constructed using BiNGO. The color depth of nodes refers to the corrected P-value of ontologies. Ontology refers to a semantic convention for a group of concepts discussed within a certain scope of knowledge. The size of nodes refers to the numbers of genes that are involved in the ontologies.

platform to reveal genes sharing common expression patterns with hub genes and to further study their associations (Fig. 5). The biological process analysis of hub genes was constructed using BiNGO. The color depth of nodes refers to the corrected $\mathrm{P}$-value of ontologies. The size of nodes refers to the numbers of genes that are involved in the ontologies. Finally, the potential biological characteristics of the co-expression network were visualized (Fig. 6).

Validation and analysis of hub genes. To validate the differences in the expression levels of the hub genes, the Oncomine database was utilized. Heat map of DEGs identified from the Oncomine database. The color depth represents the significance of the difference. TSCC vs. normal. References are as follows: 1, Tongue squamous cell carcinoma vs. Normal. Estilo Head-Neck, BMC Cancer, 2009; 2, Tongue squamous cell carcinoma vs. Normal. Kuriakose Head-Neck, Cell Mol Life Sci, 2004; 3, Tongue squamous cell carcinoma vs. Normal. Talbot Lung, Cancer Res, 2005; 4, Tongue squamous cell carcinoma vs. Normal. Ye Head-Neck. BMC Genomics, 2008. (Fig. 7). Furthermore, the overall survival time expression analysis of 11 hub genes was performed using the Kaplan-Meier plotter database. The analysis results revealed that seven hub genes exhibited a remarkable association with survival time (Figs. 8 and 9). In patients with TSCC, increased expression levels of MMP1, MMP3, FN1, SERPINE1, SPP1, MMP13 and SNAI2 were associated with a worse overall survival rate. In addition, CXCL12, MMP3, FN1, SPP1 and STAT1 were found to be differentially expressed in the various tumor stages. The expression of CXCL12, FN1, MMP3, SPP1 and STAT1 were significantly related with the tumor stage $(\mathrm{P}<0.05)$. However, the other hub genes were not significantly related with the tumor stage $(\mathrm{P}>0.05$; Fig. 10).

Prediction and enrichment analysis of miRNAs related to hub genes. To further elucidate the mechanisms of action and regulatory networks of the hub genes, miRNAs associated with hub genes were predicted (Table III). Enrichment analysis of the predicted miRNAs was subsequently performed. GO analysis indicated that miRNAs were significantly enriched in the toll-like receptor (TLR)1:TLR2 signaling pathway, nucleic acid binding transcription factor activity and cellular protein modification process. Furthermore, KEGG pathway enrichment analysis revealed that pathways associated with the TGF- $\beta$, Ras, prolactin and MAPK signaling pathways were the most enriched (Fig. 11).

Results of RT-qPCR analysis. The expression levels of SPP1 and FN1 were further measured using RT-qPCR. The results indicated that the relative expression levels of SPP1 and FN1 were 

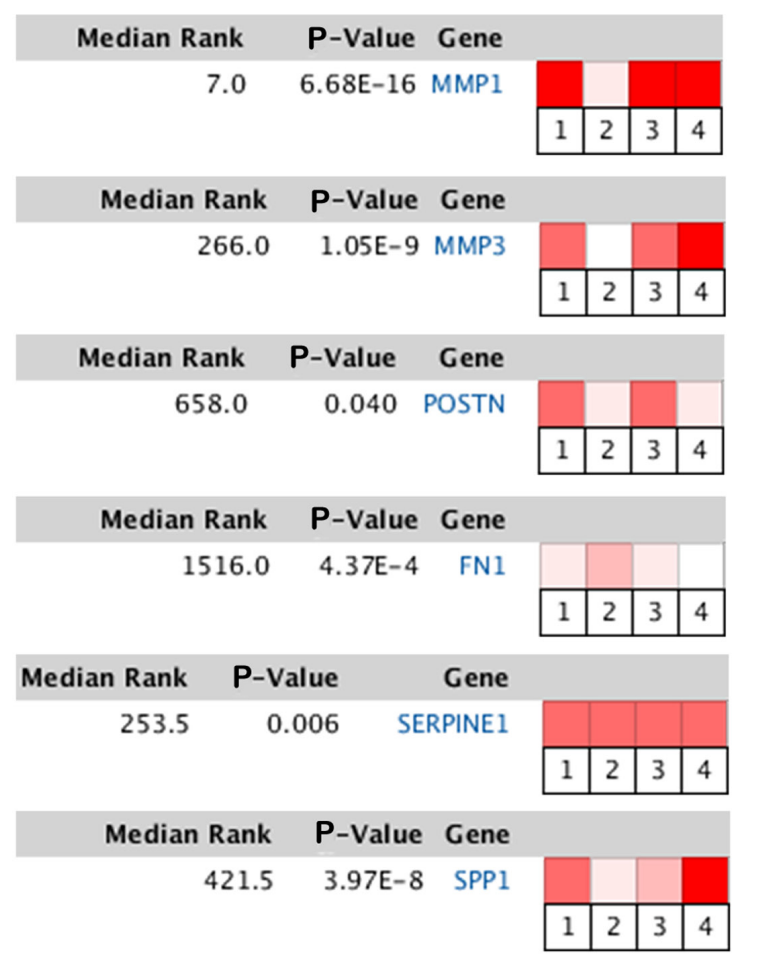

\begin{tabular}{|c|c|c|c|c|c|c|}
\hline Median Rank & P-Value & Gene & & & & \\
\hline \multirow[t]{2}{*}{805.0} & \multirow[t]{2}{*}{0.053} & \multirow[t]{2}{*}{ IGFBP3 } & & & & \\
\hline & & & 1 & 2 & 3 & 4 \\
\hline Median Rank & P-Value & Gene & & & & \\
\hline \multirow[t]{2}{*}{8231.5} & 0.999 & CXCL12 & & & & \\
\hline & & & 1 & 2 & 3 & 4 \\
\hline
\end{tabular}

\begin{tabular}{|rrr|r|r|r|r|} 
Median Rank & P-Value & Gene & \multicolumn{3}{|l|}{} \\
\hline 60.5 & $1.58 \mathrm{E}-4$ & STAT1 & \multicolumn{3}{|l|}{} & \multicolumn{3}{|c|}{} \\
\cline { 4 - 7 } & & & 2 & 2 & 3 & 4 \\
\hline
\end{tabular}

\begin{tabular}{|c|c|c|c|c|c|c|}
\hline Median Rank & P-Value & Gene & & & & \\
\hline \multirow[t]{2}{*}{597.5} & $9.55 \mathrm{E}-6$ & MMP13 & & & & \\
\hline & & & 1 & 2 & 3 & 4 \\
\hline
\end{tabular}

\begin{tabular}{|c|c|c|c|c|c|c|}
\hline Median Rank & P-Value & Gene & & & & \\
\hline \multirow[t]{2}{*}{706.0} & $3.45 E-4$ & SNAI2 & & & & \\
\hline & & & 1 & 2 & 3 & 4 \\
\hline
\end{tabular}

Figure 7. Heat map of DEGs identified from the Oncomine database. The color depth represents the significance of the difference. TSCC vs. normal. MMP1, matrix metallopeptidase 1; MMP3, matrix metallopeptidase 3; POSTN, periostin; FN1, fibronectin 1; SERPINE1, serpin family E member 1; SPP1, secreted phosphoprotein 1; IGFBP3, insulin like growth factor binding protein 3; CXCL12, C-X-C motif chemokine ligand 12; STAT1, signal transducer and activator of transcription 1; MMP13, matrix metallopeptidase 13; SNAI2, snail family transcriptional repressor 2 .

significantly increased in the TSCC tissue samples compared with those in the control group (Fig. 12). This finding suggested that SPP1 and FN1 may be considered as biomarkers for TSCC.

\section{Discussion}

The results of the present study demonstrated that SPP1 and FN1 were highly expressed in TSCC compared with normal tissues. Furthermore, the SPP1 and FN1 expression levels were gradually elevated with the increase of TSCC pathological staging. Patients with high SPP1 and FN1 expression levels exhibited poorer overall survival compared with those with decreased ones.

SPP1, also known as osteopontin, is a widely expressed viscous glycoprotein with several biological activities, which is secreted by various cell types, including osteoclasts and $\mathrm{T}$ cells (44). SPP1 may be detected in normal tissues, body fluids and cells and is involved in various physiological processes, such as development, differentiation, inflammation and wound healing (45). It has been reported that increased expression of SPP1 plays an important role in the occurrence and metastasis of various malignant tumor types (46). When tumor cells invade the ECM, SPP1 promotes the expression of MMPs in tumor cells through the NF- $\mathrm{KB}$-dependent signal transduction pathway (47). Subsequently, MMPs degrade the cell basement membrane and ECM, resulting in tumor invasion and metastasis, as well as a poor prognosis for patients with cutaneous melanoma $(47,48)$. SPP1 is also overexpressed in lung adenocarcinomas and serves as an independent prognostic biomarker, especially for T1, T2 and N0 tumor stages (44). However, the predictive value of SPP1 in lung squamous cell carcinoma needs further investigation (49). In addition, SPP1 is significantly upregulated in patients with colorectal cancer compared with healthy individuals (50). Therefore, it has been previously demonstrated that SPP1 promotes colorectal cancer metastasis by activating uncommon interstitial transformation, thus, SPP1 is considered as a potential therapeutic target for patients with colorectal cancer (51). Overexpression of SPP1 may also be involved in the development of nasopharyngeal carcinoma $(52,53)$, while a previous study has revealed that polymorphisms in the SPP1 gene are associated with other malignant tumor types such as gliomas and lung cancer (54). Zou et al (55) performed bioinformatics analysis to identify the key DEGs between oral squamous cell carcinoma (OSCC) and normal tissues. The analysis revealed that SPP1, integrin subunit $\alpha 3$ and PLAU could be the key candidate genes of OSCC (55). Furthermore, SPP1 was upregulated in OSCC, indicating that it could serve as an underlying predictor of OSCC (56). A previous study demonstrated that SPP1 was significantly associated with esophageal squamous cell carcinoma and could; therefore, be a potential target for therapy (57). Several studies have also suggested that SPP1 may be involved in the occurrence and development of OSCC (58-61). These studies indicated that SPP1 may act as an underlying biomarker of TSCC, a subtype of OSCC. The present study provided direct evidence that SPP1 expression was associated with TSCC. Therefore, the present data revealed that SPP1 was highly expressed in TSCC compared with normal tissues, while SPP1 and FN1 levels were gradually elevated with the increase in TSCC pathological staging. Compared with individuals exhibiting decreased SPP1 expression levels, upregulation of SPP1 and FN1 in patients with TSCC was associated with a poor overall survival rate. However, to the best of our knowledge, no studies have been performed to investigate the role of SPP1 in TSCC. The molecular and biological functions of SPP1 and its cancer-promoting effects in other types of cancer (62), led to the hypothesis that SPP1 also played an important role in the occurrence of TSCC. 

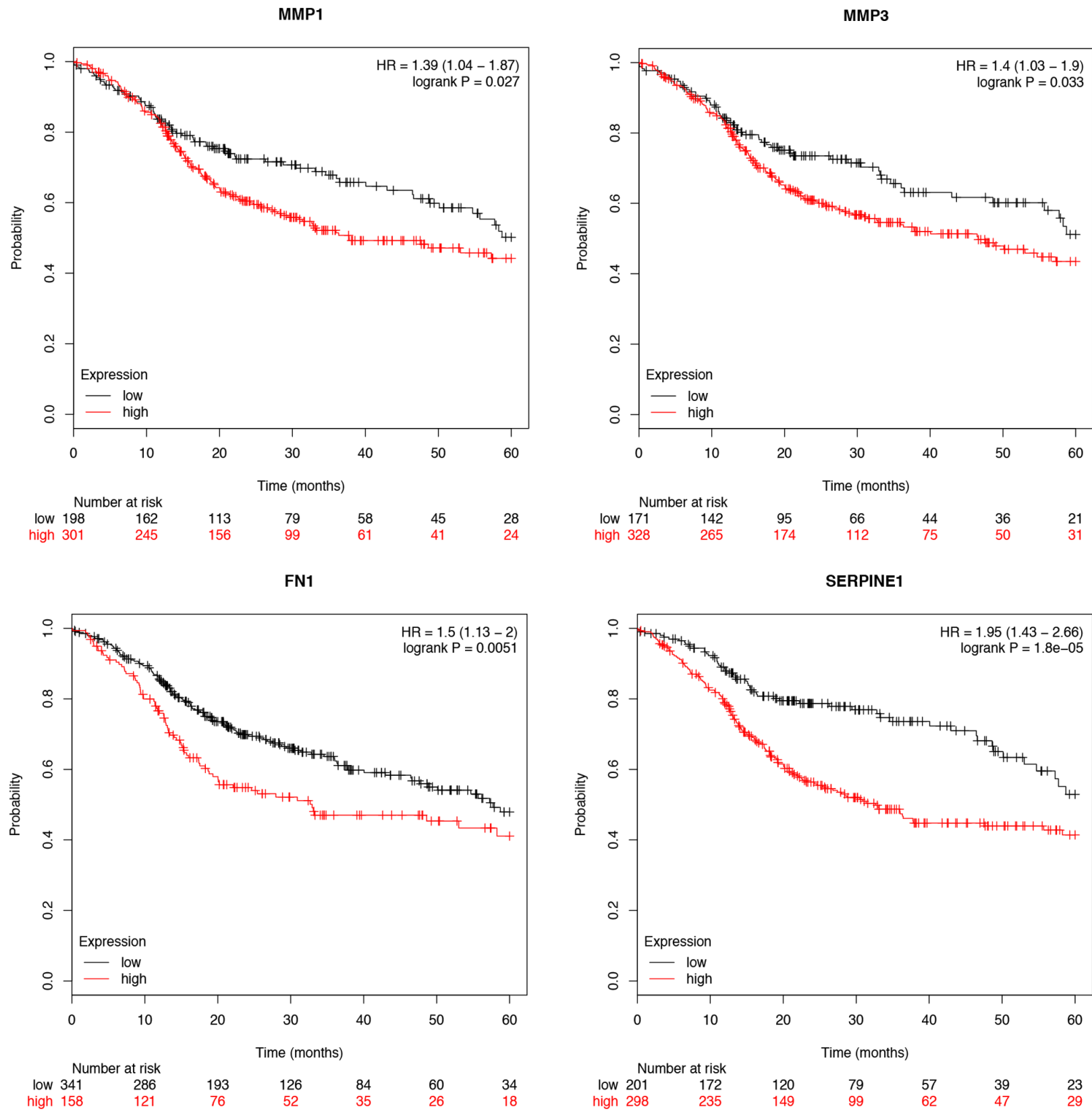

Figure 8. Overall survival analysis of the 4 hub genes, MMP1, MMP3, FN1, and SERPINE1. The high position line represented gene expression level above the median, and the low position line represented gene expression level below the median. MMP, matrix metallopeptidase; FN, fibronectin; SERPINE, serpin family E member; HR, hazard ratio.

Therefore, the clinical detection of SPP1 may be used as a diagnostic biomarker for TSCC.

FN1 is composed of a variety of homologous repeating units and participates in cell movement, growth and differentiation as well as matrix formation, while it also plays an important role in the mechanisms behind cell adhesion $(63,64)$. It has been reported that FN1 affects cell migration by mediating cell-to-cell and cell matrix adhesion (65). Overexpression of FN1 promotes adhesion and aggregation of tumor cells by affecting the movement, differentiation and growth of tumor cells (66). Furthermore, FN1 inhibits migration of tumor cells and triggers tumor metastasis through mediating intercellular and cell matrix adhesion (67). In addition, FN1, serves as a ligand for 12 members of the integrin receptor family, an important family of ECM-associated adhesion receptors (68). Morita et al (69) demonstrated that FN1 overexpression accelerates the progress and lymph node metastasis of OSCC through promoting the expression of vascular endothelial growth factor-C. A number of previous studies have provided insights into the role of FN1 as a novel biomarker for OSCC (70-72), indicating that FN1 may also be used as an underlying biomarker of TSCC. This hypothesis was further verified in the present study. FN1 affects the fourth stage of local invasion and spread of tumors, namely the migration of tumor cells (67). Tumor cells migrate through the basement membrane with an amoeboid form of movement, following the binding of the integrin transmembrane receptor to FN1 (73). FN1 also participates in matrix remodeling and affects cell movement by regulating actin aggregation (74). Therefore, investigating 
Table III. The potential microRNAs associated with the hub genes.

\section{Gene}

Predicted microRNAs

hsa-miR-558, hsa-miR-202-3p and hsa-miR-520g-5p hsa-miR-365b-3p, hsa-miR-365a-3p and hsa-miR-550b-2-5p hsa-miR-19b-3p, hsa-miR-19a-3p and hsa-miR-5590-3p hsa-miR-613, hsa-miR-1271-5p and hsa-miR-96-5p hsa-miR-6088, hsa-miR-148b-3p and hsa-miR-152-3p hsa-miR-181c-5p, hsa-miR-181a-5p and hsa-miR-181d-5p hsa-miR-19a-3p, hsa-miR-19b-3p and hsa-miR-212-5p hsa-miR-137, hsa-miR-135a-5p and hsa-miR-135b-5p hsa-miR-216a-3p, hsa-miR-3681-3p and hsa-miR-128-3p hsa-miR-27a-3p, hsa-miR-27b-3p and hsa-miR-1267 hsa-miR-124-3p.1, hsa-miR-206 and hsa-miR-1-3p

CXCL, C-X-C motif chemokine ligand; FN, fibronectin; IGFBP, insulin like growth factor binding protein; miR, microRNA; MMP, matrix metallpeptidase; POSTN, periostin; SERPINE, serpin family E member; SNAI, snail family transcriptional repressor; SPP, secreted phosphoprotein.
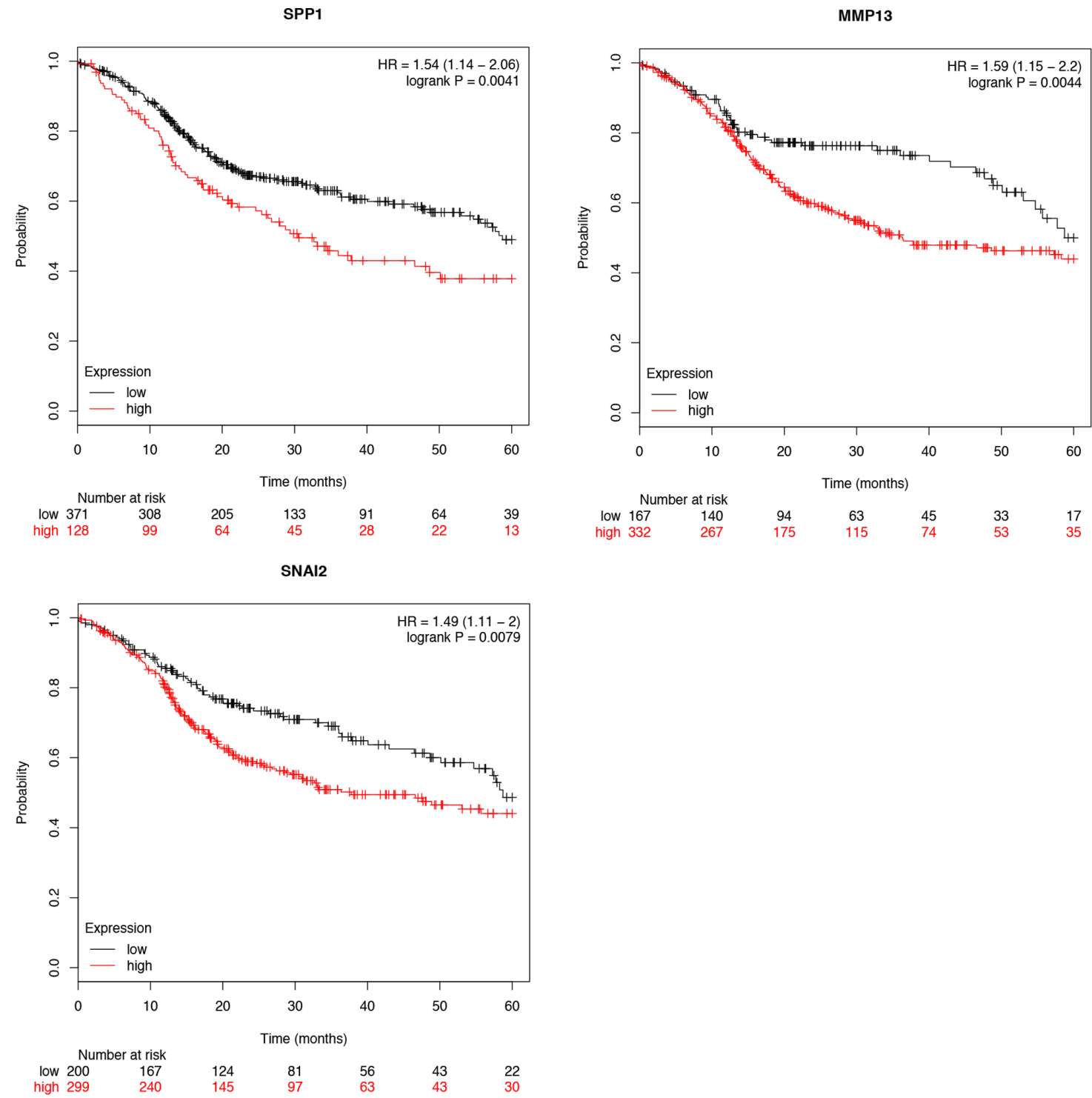

Figure 9. Overall survival analysis of the 3 hub genes, SPP1, MMP13, and SNAI2. The high position line represented gene expression level above the median, and the low position line represented gene expression level below the median. SPP, secreted phosphoprotein; MMP, matrix metallopeptidase; SNAI, snail family transcriptional repressor; HR, hazard ratio. 

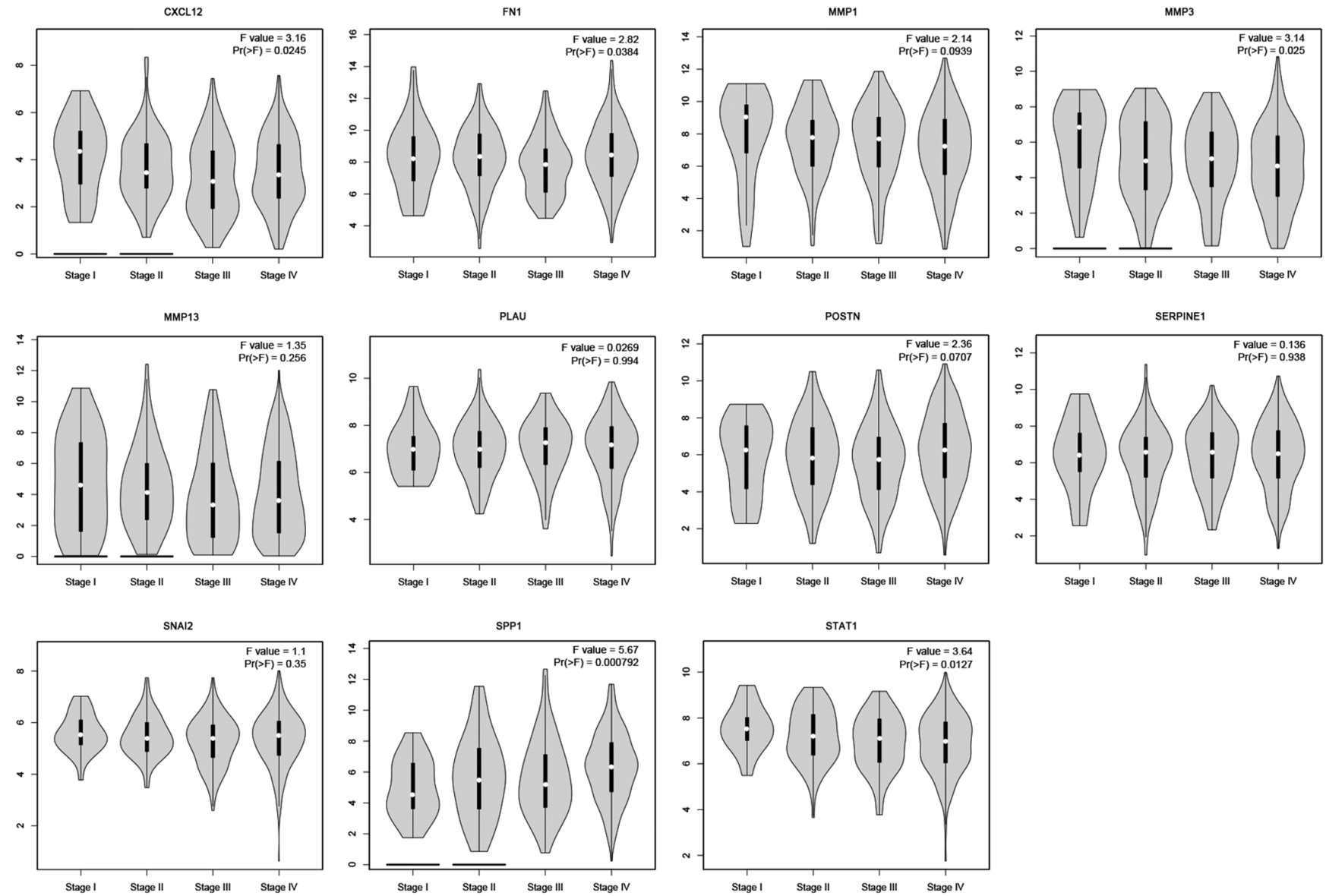

Figure 10. Hub genes expressed differentially in each tumor stage. The expression of CXCL12, FN1, MMP3, SPP1 and STAT1 were significantly different in different tumor stages. However, MMP1, MMP13, PLAU, POSTN, SERPINE1 and SNAI2 were not significantly related with the tumor stage (P>0.05). CXCL, C-X-C motif chemokine ligand; FN, fibronectin; MMP, matrix metallopeptidase; PLAU, plasminogen activator urokinase; POSTN, periostin; SERPINE, serpin family E member; SNAI, snail family transcriptional repressor; SPP, secreted phosphoprotein; STAT, signal transducer and activator of transcription.

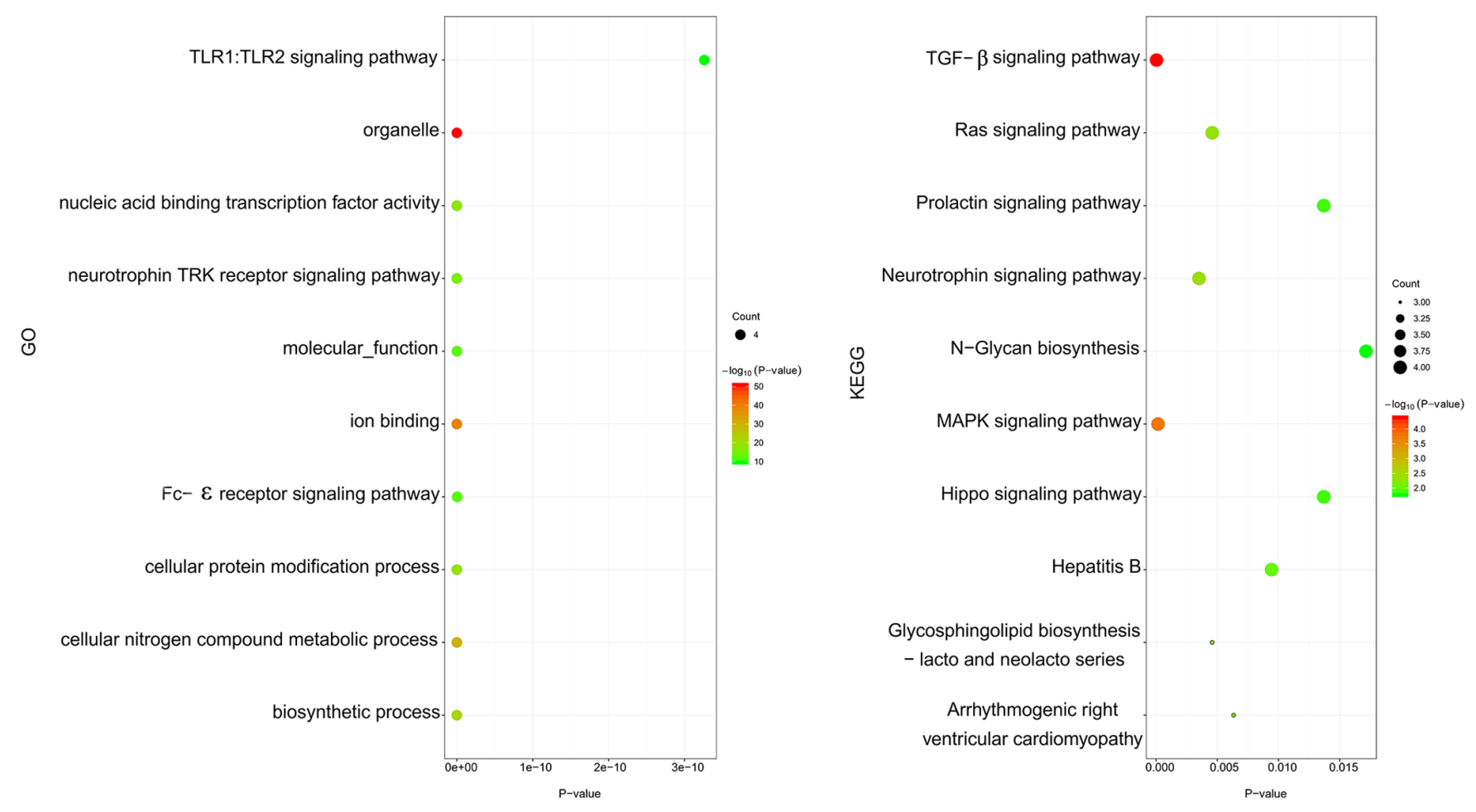

Figure 11. GO and KEGG enrichment analysis of predicted miRNAs associated to hub genes. The larger the diameter of the circle, the more genes were enriched in that item. The redder the circle, the smaller the P-value. TLR, toll-like receptor; TRK, tyrosine kinase. 

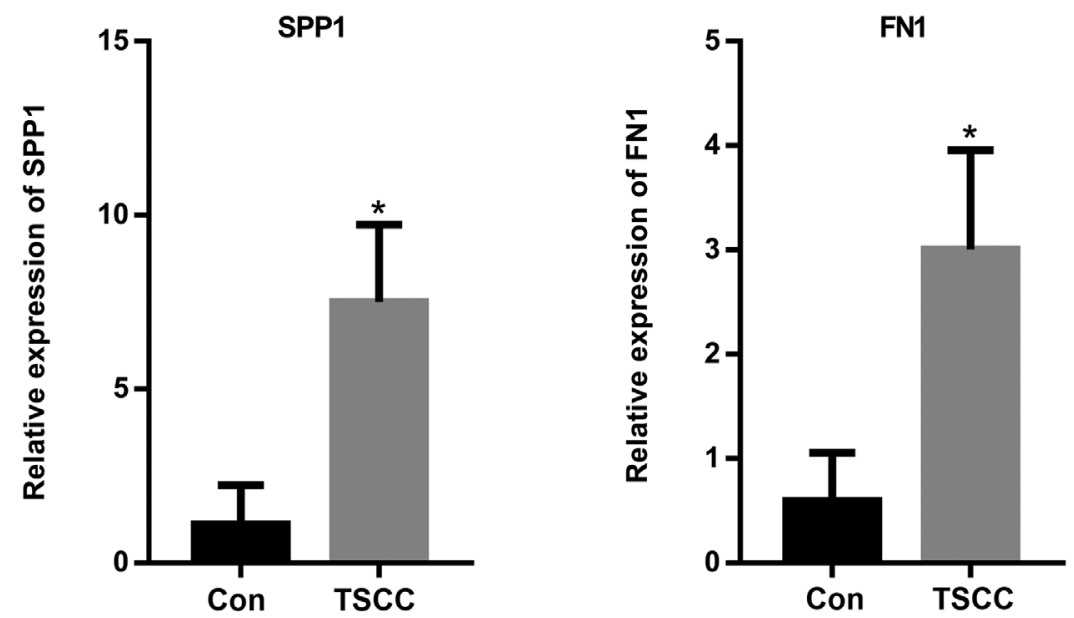

Figure 12. Relative expression levels of SPP1 and FN1 as determined using reverse transcription-quantitative PCR. *P<0.05 vs. Con. Con, control; FN1, fibronectin; TSCC, tongue squamous cell carcinoma; SPP, secreted phosphoprotein.

the interactions and mechanisms through which FN1 functions may improve the understanding of its role in TSCC, provide novel approaches for investigating its underlying mechanisms of action and develop more effective treatments. Studying the molecular mechanism of action of FN1 behind the occurrence and progression of TSCC may further the current understanding of the potential targeting of FN1 for the treatment of TSCC (70).

It should be noted that the present study has some limitations. Firstly, this was an observational study based on the bioinformatics analysis of 11 key genes. Therefore, the results of the present study provided novel clues that could be used for subsequent, in-depth studies investigating the developmental mechanisms of action behind TSCC. In addition, future studies involving more samples should be conducted to verify the results of the present study.

In conclusion, a total of 83 DEGs and 11 hub genes, especially SPP1 and FN1, were obtained from the bioinformatics and microarray assays between TSCC and normal tissues. These genes could be used as diagnostic and therapeutic biomarkers for TSCC. Furthermore, SPP1 and FN1 may be associated with the occurrence, lymph node metastasis and malignant progression of TSCC. Therefore, both molecules were considered as potential biomarkers for monitoring TSCC progression.

\section{Acknowledgements}

The authors would like to thank Dr. Peng Guo (The Fourth Hospital of Hebei Medical University, Shijiazhuang, China) for his statistical assistance and suggestions during the submitting process.

\section{Funding}

No funding was received.

\section{Availability of data and materials}

The datasets used and/or analyzed during the current study are available from the corresponding author on reasonable request.

\section{Authors' contributions}

XLX and HL performed the experiments and were major contributors in writing the manuscript and submitting the manuscript. TKL made substantial contributions to research conception and designed the draft of the research process. $\mathrm{YZ}$ and SXZ were involved in revising manuscript critically for important intellectual content and made substantial contributions to conception and design, acquisition of data, and analysis and interpretation of all data. ZC and YB analyzed the data. YZ and SXZ confirmed the authenticity of all the raw data. All authors read and approved the final manuscript.

\section{Ethics approval and consent to participate}

The data of this research was downloaded from the GEO database, a public database. All institutional and national guidelines for the care and use of participates were followed. The research conformed to the standards set by the Declaration of Helsinki and was authorized by the Human Ethics and Research Ethics Committees of the Fourth Hospital of the Hebei Medical University. Written informed consents were obtained from all participants.

\section{Patient consent for publication}

Not applicable.

\section{Competing interests}

The authors declare that they have no competing interests.

\section{References}

1. Paderno A, Morello R and Piazza C: Tongue carcinoma in young adults: A review of the literature. Acta Otorhinolaryngol Ital 38: 175-180, 2018.

2. Mannelli G, Arcuri F, Agostini T, Innocenti M, Raffaini M and Spinelli G: Classification of tongue cancer resection and treatment algorithm. J Surg Oncol 117: 1092-1099, 2018.

3. Sun L, Liang J, Wang Q, Li Z, Du Y and Xu X: MicroRNA-137 suppresses tongue squamous carcinoma cell proliferation, migration and invasion. Cell Prolif 49: 628-635, 2016. 
4. Semsettin B, Sinan E and Nigar V: Comparison of the effects of topical cyclosporine a $0.05 \%$, cyclosporine a $2 \%$, epinastine hydrochloride $0.05 \%$, and prednisolone acetate $1 \%$ on allergic inflammation in an experimental allergic conjunctivitis model. Cornea 32: 1465-1469, 2013

5. Bello IO, Soini Y and Salo T: Prognostic evaluation of oral tongue cancer: Means, markers and perspectives (II). Oral Oncol 46: 636-643, 2010.

6. Ahmadi N, Chan M, Huo YR, Sritharan N and Chin RY: Survival outcome of tonsillar squamous cell carcinoma (TSCC) in the context of human papillomavirus (HPV): A systematic review and meta-analysis. Surgeon 17: 6-14, 2019.

7. Ramqvist T, Grün N and Dalianis T: Human papillomavirus and tonsillar and base of tongue cancer. Viruses 7: 1332-1343, 2015.

8. Lozev I, Ruseva S, Pidakev I, Cardoso JC, Wollina U, Lotti T, Maximov GK, Terziev I and Tchernev G: Mucoepidermoid carcinoma (MEC) of parotid gland with massive cutaneous involvement: Bilateral pedicle advancement flap (U-Plasty) as adequate surgical approach. Open Access Maced J Med Sci 6: 134-136, 2018.

9. Zhu L, Wang Y, Li R, Liu A, Zhang X, Zuo C and Xu X: Surgical treatment of early tongue squamous cell carcinoma and patient survival. Oncol Lett 17: 5681-5685, 2019.

10. Ng JH, Iyer NG, Tan MH and Edgren G: Changing epidemiology of oral squamous cell carcinoma of the tongue: A global study. Head Neck 39: 297-304, 2017.

11. Wade MH and Plotnick H: Xeroderma pigmentosum and squamous cell carcinoma of the tongue. Identification of two black patients as members of complementation group C. J Am Acad Dermatol 12: 515-521, 1985.

12. Zhang P, Zhang L, Liu H, Zhao L, Li Y, Shen JX, Liu Q, Liu MZ and Xi M: Clinicopathologic characteristics and prognosis of tongue squamous cell carcinoma in patients with and without a history of radiation for nasopharyngeal carcinoma: A matched case-control study. Cancer Res Treat 49: 695-705, 2017.

13. Velly AM, Franco EL, Schlecht N, Pintos J, Kowalski LP, Oliveira BV and Curado MP: Relationship between dental factors and risk of upper aerodigestive tract cancer. Oral Oncol 34: 284-291, 1998.

14. Troiano G, Rubini C, Togni L, Caponio VC, Zhurakivska K, Santarelli A, Cirillo N, Lo Muzio L and Mascitti M: The immune phenotype of tongue squamous cell carcinoma predicts early relapse and poor prognosis. Cancer Med 9: 8333-8344, 2020.

15. Hussein A A, Forouzanfar T, Bloemena E, de Visscher J, Brakenhoff RH, Leemans CR and Helder MN: A review of the most promising biomarkers for early diagnosis and prognosis prediction of tongue squamous cell carcinoma. Br J Cancer 119: 724-736, 2018

16. Nakamura K, Akiba J, Ogasawara S, Naito Y, Nakayama M, Abe Y, Kusukawa J and Yano H: SUOX is negatively associated with multistep carcinogenesis and proliferation in oral squamous cell carcinoma. Med Mol Morphol 51: 102-110, 2018.

17. Zheng G, Zhang Z, Liu H, Xiong Y, Luo L, Jia X, Peng C Zhang Q, Li N, Gu Y, et al: HSP27-mediated extracellular and intracellular signaling pathways synergistically confer chemoresistance in squamous cell carcinoma of tongue. Clin Cancer Res 24: 1163-1175, 2018.

18. Chen H and Dai J: miR-409-3p suppresses the proliferation, invasion and migration of tongue squamous cell carcinoma via targeting RDX. Oncol Lett 16: 543-551, 2018

19. Solomon B, Young RJ and Rischin D: Head and neck squamous cell carcinoma: Genomics and emerging biomarkers for immunomodulatory cancer treatments. Semin Cancer Biol 52 228-240, 2018

20. Falzone L, Lupo G, La Rosa G, Crimi S, Anfuso CD, Salemi R, Rapisarda E, Libra M and Candido S: Identification of nove MicroRNAs and their diagnostic and prognostic significance in oral cancer. Cancers (Basel) 11: 610, 2019.

21. Yu M, Wu G, Chen Y, Wang H, Gao Y and Wang A: Bioinformatic screening and experimental analysis identify SFRP1 as a prognostic biomarker for tongue squamous cell carcinomas. Arch Oral Biol 110: 104587, 2020

22. Wang R, Zhou X, Wang H, Zhou B, Dong S, Ding Q, Peng M, Sheng X, Yao J, Huang R, et al: Integrative analysis of gene expression profiles reveals distinct molecular characteristics in ora tongue squamous cell carcinoma. Oncol Lett 17: 2377-2387, 2019.

23. Usami Y, Ishida K, Sato S, Kishino M, Kiryu M, Ogawa Y, Okura M, Fukuda Y and Toyosawa S: Intercellular adhesion molecule-1 (ICAM-1) expression correlates with oral cancer progression and induces macrophage/cancer cell adhesion. Int J Cancer 133: 568-578, 2013
24. Zhang D, Chen ZG, Liu SH, Dong ZQ, Dalin M, Bao SS, Hu YW and Wei FC: Galectin-3 gene silencing inhibits migration and invasion of human tongue cancer cells in vitro via downregulating $\beta$-catenin. Acta Pharmacol Sin 34: 176-184, 2013.

25. Wang C, Liu X, Chen Z, Huang H, Jin Y, Kolokythas A, Wang A, Dai Y, Wong DT and Zhou X: Polycomb group protein EZH2-mediated E-cadherin repression promotes metastasis of oral tongue squamous cell carcinoma. Mol Carcinog 52: 229-236, 2013.

26. Candido S, Lupo G, Pennisi M, Basile MS, Anfuso CD, Petralia MC, Gattuso G, Vivarelli S, Spandidos DA, Libra M and Falzone L: The analysis of miRNA expression profiling datasets reveals inverse microRNA patterns in glioblastoma and Alzheimer's disease. Oncol Rep 42: 911-922, 2019.

27. Falzone L, Romano GL, Salemi R, Bucolo C, Tomasello B, Lupo G, Anfuso CD, Spandidos DA, Libra M and Candido S: Prognostic significance of deregulated microRNAs in uveal melanomas. Mol Med Rep 19: 2599-2610, 2019.

28. Falzone L, Scola L, Zanghì A, Biondi A, Di Cataldo A, Libra M and Candido $\mathrm{S}$ : Integrated analysis of colorectal cancer microRNA datasets: Identification of microRNAs associated with tumor development. Aging (Albany NY) 10: 1000-1014, 2018.

29. Falzone L, Candido S, Salemi R, Basile MS, Scalisi A, McCubrey JA, Torino F, Signorelli SS, Montella M and Libra M: Computational identification of microRNAs associated to both epithelial to mesenchymal transition and NGAL/MMP-9 pathways in bladder cancer. Oncotarget 7: 72758-72766, 2016.

30. Hafsi S, Candido S, Maestro R, Falzone L, Soua Z, Bonavida B, Spandidos DA and Libra M: Correlation between the overexpression of Yin Yang 1 and the expression levels of miRNAs in Burkitt's lymphoma: A computational study. Oncol Lett 11: 1021-1025, 2016

31. Li R, Faden DL, Fakhry C, Langelier C, Jiao Y, Wang Y, Wilkerson MD, Pedamallu CS, Old M, Lang J, et al: Clinical, genomic, and metagenomic characterization of oral tongue squamous cell carcinoma in patients who do not smoke. Head Neck 37: 1642-1649, 2015

32. Li B, Li CH, Guo H, Chen J and Wang SX: Analysis of 27 cases of defect restoration using infrahyoid myocutaneous flap after intraoral cancer surgery. Zhonghua Er Bi Yan Hou Tou Jing Wai Ke Za Zhi 43: 826-829, 2008 (In Chinese).

33. Liu X, Qiao B, Zhao T, Hu F, Lam AK and Tao Q: Sox 2 promotes tumor aggressiveness and epithelial-mesenchymal transition in tongue squamous cell carcinoma. Int J Mol Med 42: 1418-1426, 2018.

34. Tang Q, Cheng B, Xie M, Chen Y, Zhao J, Zhou X and Chen L: Circadian clock gene bmall inhibits tumorigenesis and increases paclitaxel sensitivity in tongue squamous cell carcinoma. Cancer Res 77: 532-544, 2017.

35. Tanaka Y, Araki K, Tanaka S, Miyagawa Y, Suzuki H, Kamide D, Tomifuji M, Uno K, Kimura E, Yamashita T, et al: Sentinel lymph node-targeted therapy by oncolytic sendai virus suppresses micrometastasis of head and neck squamous cell carcinoma in an orthotopic nude mouse model. Mol Cancer Ther 18: 1430-1438, 2019.

36. Xiong J, Feng J, Qiu L, Gao Z, Li P, Pang L and Zhang Z: SDF-1-loaded PLGA nanoparticles for the targeted photoacoustic imaging and photothermal therapy of metastatic lymph nodes in tongue squamous cell carcinoma. Int J Pharm 554: 93-104, 2019.

37. McCubrey JA, Lertpiriyapong K, Steelman LS, Abrams SL, Yang LV, Murata RM, Rosalen PL, Scalisi A, Neri LM, Cocco L, et al: Effects of resveratrol, curcumin, berberine and other nutraceuticals on aging, cancer development, cancer stem cells and microRNAs. Aging (Albany NY) 9: 1477-1536, 2017.

38. Qiu Y, Meng LB, Di CY, Huo YH, Yao BC, Zhang TJ and Hua Z: Exploration of the differentially expressed long noncoding RNAs and genes of morphine tolerance via bioinformatic analysis. J Comput Biol 26: 1379-1393, 2019.

39. Zou YF, Meng LB, Wang QQ, He ZK, Hu CH, Shan MJ, Wang DY and $\mathrm{Yu} X$ : Identification and functional enrichment analysis of potential diagnostic and therapeutic targets in adamantinomatous craniopharyngioma. J Comput Biol 27: 55-68, 2019.

40. Zhang H, Liu J, Fu X and Yang A: Identification of key genes and pathways in tongue squamous cell carcinoma using bioinformatics analysis. Med Sci Monit 23: 5924-5932, 2017.

41. Li T, Wu Q, Liu D and Wang X: miR-27b suppresses tongue squamous cell carcinoma epithelial-mesenchymal transition by targeting ITGA5. Onco Targets Ther 13: 11855-11867, 2020. 
42. Chen Y, Guo Y and Yan W: lncRNA RP5-916L7.2 correlates with advanced tumor stage, and promotes cells proliferation while inhibits cells apoptosis through targeting miR-328 and miR-939 in tongue squamous cell carcinoma. Clin Biochem 67: 24-32, 2019.

43. Meng LB, Shan MJ, Qiu Y, Qi R, Yu ZM, Guo P, Di CY and Gong T: TPM2 as a potential predictive biomarker for atherosclerosis. Aging (Albany NY) 11: 6960-6982, 2019.

44. Zhang Y, Du W, Chen Z and Xiang C: Upregulation of PD-L1 by SPP1 mediates macrophage polarization and facilitates immune escape in lung adenocarcinoma. Exp Cell Res 359: 449-457, 2017.

45. Kramerova I, Kumagai-Cresse C, Ermolova N, Mokhonova E, Marinov M, Capote J, Becerra D, Quattrocelli M, Crosbie RH, Welch E, et al: Spp1 (osteopontin) promotes TGF $\beta$ processing in fibroblasts of dystrophin-deficient muscles through matrix metalloproteinases. Hum Mol Genet 28: 3431-3442, 2019.

46. Morse C, Tabib T, Sembrat J, Buschur KL, Bittar HT, Valenzi E, Jiang Y, Kass DJ, Gibson K, Chen W, et al: Proliferating SPP1/MERTK-expressing macrophages in idiopathic pulmonary fibrosis. Eur Respir J 54: 1802441, 2019.

47. Guarneri C, Bevelacqua V, Polesel J, Falzone L, Cannavò PS, Spandidos DA, Malaponte G and Libra M: NF- $\kappa$ B inhibition is associated with OPN/MMP-9 downregulation in cutaneous melanoma. Oncol Rep 37: 737-746, 2017.

48. Shevde LA and Samant RS: Role of osteopontin in the pathophysiology of cancer. Matrix Biol 37: 131-141, 2014.

49. Li S, Yang R, Sun X, Miao S, Lu T, Wang Y, Wo Y and Jiao W: Identification of SPP1 as a promising biomarker to predict clinical outcome of lung adenocarcinoma individuals. Gene 679: 398-404, 2018

50. Choe EK, Yi JW, Chai YJ and Park KJ: Upregulation of the adipokine genes ADIPOR1 and SPP1 is related to poor survival outcomes in colorectal cancer. J Surg Oncol 117: 1833-1840, 2018.

51. Xu C, Sun L, Jiang C, Zhou H, Gu L, Liu Y and Xu Q: SPP1, analyzed by bioinformatics methods, promotes the metastasis in colorectal cancer by activating EMT pathway. Biomed Pharmacother 91: 1167-1177, 2017.

52. Wang HH, Wang XW and Tang CE: Osteopontin expression in nasopharyngeal carcinoma: Its relevance to the clinical stage of the disease. J Cancer Res Ther 7: 138-142, 2011.

53. Ma R, Luo X, Feng S, Li J, Fan Y, Wen W and Li H: Osteopontin promotes EZH2 expression and tumor progression in nasopharyngeal carcinoma. ORL J Otorhinolaryngol Relat Spec 76 273-281, 2014

54. Yang G, Peng X, Guo P and Yang G: Association of osteopontin polymorphism with cancer risk: A meta-analysis. Int J Clin Exp Med 8: 20911-20917, 2015.

55. Zou B, Li J, Xu K, Liu JL, Yuan DY, Meng Z and Zhang B: Identification of key candidate genes and pathways in oral squamous cell carcinoma by integrated Bioinformatics analysis. Exp Ther Med 17: 4089-4099, 2019.

56. Zhang C, Man DP, Ma SM, Cao SW and Li DW: Expressions and significances of CD147, OPN and MMP-2 in oral squamous cell carcinoma. Sichuan Da Xue Xue Bao Yi Xue Ban 43: 683-686, 2012 (In Chinese).

57. Ito T, Hashimoto Y, Tanaka E, Kan T, Tsunoda S, Sato F, Higashiyama M, Okumura T and Shimada Y: An inducible short-hairpin RNA vector against osteopontin reduces metastatic potential of human esophageal squamous cell carcinoma in vitro and in vivo. Clin Cancer Res 12: 1308-1316, 2006.

58. Hu Q, Peng J, Chen X, Li H, Song M, Cheng B and Wu T: Obesity and genes related to lipid metabolism predict poor survival in oral squamous cell carcinoma. Oral Oncol 89: 14-22, 2019.

59. Zhang X, Zhang L, Tan X, Lin Y, Han X, Wang H, Ming H, Li Q, Liu K and Feng G: Systematic analysis of genes involved in oral cancer metastasis to lymph nodes. Cell Mol Biol Lett 23: 53, 2018.
60. D'Addazio G, Artese L, Traini T, Rubini C, Caputi S and Sinjari B Immunohistochemical study of osteopontin in oral squamous cell carcinoma allied to fractal dimension. J Biol Regul Homeost Agents 32: 1033-1038, 2018.

61. Huang CF, Yu GT, Wang WM, Liu B and Sun ZJ: Prognostic and predictive values of SPP1, PAI and caveolin-1 in patients with oral squamous cell carcinoma. Int J Clin Exp Pathol 7: 6032-6039, 2014.

62. Wang Y, Su J, Wang Y, Fu D, Ideozu JE, Geng H, Cui Q, Wang C, Chen R, Yu Y, et al: The interaction of YBX1 with G3BP1 promotes renal cell carcinoma cell metastasis via YBX1/G3BP1-SPP1- NF- $\kappa$ B signaling axis. J Exp Clin Cancer Res 38: 386, 2019.

63. Aota Y, An HS, Homandberg G, Thonar EJ, Andersson GB, Pichika R and Masuda K: Differential effects of fibronectin fragment on proteoglycan metabolism by intervertebral disc cells: A comparison with articular chondrocytes. Spine (Phila Pa 1976) 30: 722-728, 2005.

64. Beumer S, Heijnen HF, IJsseldijk MJ, Orlando E, de Groot PG and Sixma JJ: Platelet adhesion to fibronectin in flow: The importance of von Willebrand factor and glycoprotein Ib. Blood 86: 3452-3460, 1995.

65. Filenius S, Tervo T and Virtanen I: Production of fibronectin and tenascin isoforms and their role in the adhesion of human immortalized corneal epithelial cells. Invest Ophthalmol Vis Sci 44: 3317-3325, 2003.

66. Amary F, Perez-Casanova L, Ye H, Cottone L, Strobl AC, Cool P, Miranda E, Berisha F, Aston W, Rocha M, et al: Synovial chondromatosis and soft tissue chondroma: Extraosseous cartilaginous tumor defined by FN1 gene rearrangement. Mod Pathol 32: 1762-1771, 2019.

67. Cai X, Liu C, Zhang TN, Zhu YW, Dong X and Xue P: Down-regulation of FN1 inhibits colorectal carcinogenesis by suppressing proliferation, migration, and invasion. J Cell Biochem 119: 4717-4728, 2018.

68. Pankov R and Yamada KM: Fibronectin at a glance. J Cell Sci 115: 3861-3863, 2002

69. Morita Y, Hata K, Nakanishi M, Omata T, Morita N, Yura Y, Nishimura R and Yoneda T: Cellular fibronectin 1 promotes VEGF-C expression, lymphangiogenesis and lymph node metastasis associated with human oral squamous cell carcinoma. Clin Exp Metastasis 32: 739-753, 2015.

70. Yen CY, Huang CY, Hou MF, Yang YH, Chang CH, Huang HW, Chen $\mathrm{CH}$ and Chang HW: Evaluating the performance of fibronectin 1 (FN1), integrin $\alpha 4 \beta 1$ (ITGA4), syndecan-2 (SDC2), and glycoprotein CD44 as the potential biomarkers of oral squamous cell carcinoma (OSCC). Biomarkers 18: 63-72, 2013.

71. Suresh A, Vannan M, Kumaran D, Gümüs ZH, Sivadas P, Murugaian EE, Kekatpure V, Iyer S, Thangaraj K and Kuriakose MA: Resistance/response molecular signature for oral tongue squamous cell carcinoma. Dis Markers 32: 51-64, 2012.

72. Zhang Z, Pan J, Li L, Wang Z, Xiao W and Li N: Survey of risk factors contributed to lymphatic metastasis in patients with oral tongue cancer by immunohistochemistry. J Oral Pathol Med 40: 127-134, 2011.

73. Brinkhof B, Zhang B, Cui Z, Ye H and Wang H: ALCAM (CD166) as a gene expression marker for human mesenchymal stromal cell characterisation. Gene X 5: 100031, 2020.

74. Zhan S, Li J, Wang T and Ge W: Quantitative proteomics analysis of sporadic medullary thyroid cancer reveals FN1 as a potential novel candidate prognostic biomarker. Oncologist 23: 1415-1425, 2018.

This work is licensed under a Creative Commons Attribution-NonCommercial-NoDerivatives 4.0 International (CC BY-NC-ND 4.0) License. 